\section{Symbolisierungen des Bösen in der Jugendkultur}

Heinz Streib

Symbolisation of Evil in Youth Culture. The media topic of satanism as a trend in youth culture is distinguished from the young people's subjective experience of evil. Case studies are presented and discussed in various perspectives. P. Tillich's notion of the demonic help to widen the understanding of such symbolisations.

\section{Phänomenbereiche jugendkultureller Symbolisierung des Bösen}

Die Jugendkultur scheint nicht gerade arm an Einfällen zur Symbolisierung des Bösen. Wohl am auffälligsten - und darum auch meist spontan assoziiert - sind Symbolisierungen, die mit Satan zu tun haben. Satanistische Symbole finden sich in der Werbung für Musik-CDs und für Filme; sie tauchen in Videoclips zur visuellen Untermalung entsprechender Musik auf. Man kann satanistische Symbole leicht in digitaler Form im Internet finden. Es gibt sie als T-Shirt-Aufdruck zu erwerben oder auch als Kuscheltier. Mit Satanistischem, so scheint es, lässt sich gutes Geld verdienen, lassen sich Auflagen steigern. Auch Jugendzeitschriften berichten in regelmäßigen Abständen über satanistische Themen.

Nicht zuletzt mit dem Verkaufserfolg wird auch der Bekanntheitsgrad des "Satanismus" zusammenhängen. In der heutigen Generation von Jugendlichen besitzen die Vorstellungen vom Teufel und den Dämonen eine hohe Faszination und Glaubwürdigkeit. ${ }^{1}$ Wie bereits vor 20 Jahren zum spektakulären Phänomen der sog. schwarzen Messen erhoben wurde, weisen satanistische Symbole und Rituale sehr hohe Bekanntheitswerte von ca. zwei Dritteln der Jugendlichen auf, ${ }^{2}$ auch wenn aktives Praktizieren - vermutlich deutlich - unter $3 \%$ liegen dürfte. ${ }^{3}$ Satanismus und alles, was an Ritualen und (schwarz-) magischem Denken damit in Zusammenhang gebracht wird (bis hin zu Todesankündigungen per email), so berichten Lehrerinnen und Lehrer, ist bei einem Teil der Jugendlichen wohl bekannt und immer noch aktuell.

'Dem Satz „Ich glaube, den Teufel gibt es wirklich" stimmen von 1754 zum Okkultismus befragten Jugendlichen $24 \% \mathrm{zu}$ (vgl. Johannes Mischo, Abschlußbericht der Fragebogenuntersuchung bei 1754 Schülerinnen und Schülern in Rheinland-Pfalz zum Thema ,Erfahrungen Jugendlicher mit Okkultpraktiken', Freiburg i. Br. 1990).

Johannes Mischo, Okkultismus bei Jugendlichen. Ergebnisse einer empirischen Untersuchung Johannes Mischo, Okkultismus bei Jugendlichen. Ergebnisse einer empirischen Untersuchung, Untersuchungen 1989-1991, München 1993.

Anton A. Bucher, Ist Okkultismus die neue Jugendreligion? Eine empirische Untersuchung an 650 Jugendlichen, in: Archiv für Religionspsychologie 21/1994, Göttingen, 248-266.
Jugendkulturelle Symbolisierungen des Bösen kann man auch über den Bereich hinaus erkennen, in dem der Teufel und seine Dämonen explizit als Personen vorkommen. Symbolisierungen des Bösen als geheimnisvolle böse und bedrohliche Macht, die von Menschen Besitz ergreifen kann und angesichts derer sich der Held bewähren muss, finden sich auch in Video-Clips, Horrorfilmen und Gewalt-Filmen, selbst in Filmserien für Kinder und Jugendliche sowie in Computerspielen.

Angesichts dieses ersten Einblicks in den Phänomenbereich legt es sich nahe, von den medialen die subjektiven Symbolisierungen des Bösen zu unterscheiden. Mediale Symbolisierungen des Bösen werden von der Medienindustrie und anderen, die in der Jugendkultur Geld verdienen wollen, aufgegriffen und für die Jugendlichen in Szene gesetzt; sie sind grell, schreiend, plakativ und müssen dies auch sein, wenn sie Kauf- und Konsumlust ansprechen sollen. "Jugendkultur" würde jedoch, wenn diese allein auf Unterhaltungs-Massenmedien und industriell gefertigte Symbolisierungen fokussiert würde, verstanden als medial geprägte, wenn nicht gar medial dominierte Kultur. Und dies wäre ein einseitiges Bild.

Subjektive Symbolisierungen des Bösen sind solche, die von den Jugendlichen selbst konstruiert oder in die eigene Vorstellungswelt verankert werden, sodass sie in Zeichnungen, wie sie zuweilen am Rande langweiliger Unterrichtsstunden entstehen, in peer-group-Erzählungen auf dem Schulhof oder in okkulten oder satanistischen Praktiken eine Rolle spielen. Freilich sind die subjektiven ohne die medialen Symbolisierungen kaum - und angesichts der fortschreitenden Medialisierung der Jugendkultur immer weniger - denkbar; und der Übergang zwischen beiden ist fließend. Aber beides sollte unterschieden werden. Dies nicht zuletzt deswegen, weil PastoraltheologInnen und ReligionspädagogInnen keine falschen Schlüsse ziehen sollten und etwa vom Pentagramm auf dem T-Shirt oder dem Besitz von CDs mit satanischer Symbolik auf handfeste satanistische Weltbilder oder gar auf satanistisches Praktizieren schließen. ${ }^{4}$

Nicht zuletzt im Interesse pastoraler und religionspädagogischer Begleitung der Jugendlichen wird sich unser primäres Interesse auf die subjektiven Symbolisierungen des Bösen richten - und dabei auch den Inszenierungen und Ritualisierungen Beachtung schenken, die Jugendliche sich aneignen oder selbst erfinden. Die Empfehlung wäre hier - und dies war auch der Weg unserer eigenen empirischen Forschung ${ }^{5}$-, sich möglichst eng an die biographische Entwicklungsdynamik anzuschmiegen, möglichst dicht an den subjektiven Symbolisierungen der Jugendlichen zu bleiben. ${ }^{4}$ Vgl. Werner Helsper, Okkultismus - die neue Jugendreligion. Die Symbolik des Todes und des
Bösen in der Jugendkultur, Opladen 1992.

Vgl. Heinz Streib / Albrecht Schöll, Wege der Entzauberung. Jugendliche Sinnsuche und Okkultfaszination - Kontexte und Analysen, Schriften aus dem Comenius-Institut, Münster 2000; H. Zinser (Anm. 2). 


\section{Fallbeispiele jugendkultureller Symbolisierung des Bösen}

Aus unserer empirischen Forschung können eine ganze Reihe von Fällen vorgestellt werden, in denen Symbolisierungen des Bösen eine Rolle spielen und zugleich deren lebensgeschichtliche Verwurzelung sowie die gegenwärtige Psychodynamik in der Adoleszenz anschaulich werden. Darunter ragen besonders die Fälle heraus, die wir jenem unserer drei Typen zugeordnet haben, den wir „Die Geister, die ich rief ..." genannt haben. ${ }^{6}$ An zwei Fällen, Hei$\mathrm{ke}^{7}$ und Bernd ${ }^{8}$, können die lebensgeschichtliche Verwurzelung der Symbolisierungen des Bösen sowie die Motivlage und Dynamik der Faszination des Bösen gut aufgezeigt werden. Dabei zeigen sich auch markante Unterschiede zwischen einer aggressiven und einer defensiven Symbolisierung des Bösen (was vielleicht auf geschlechtspezifische Differenzen hinweisen könnte).

Heike (22), evangelisch-methodistisch getauft und in einer evangelischen Kirchengemeinde konfirmiert, berichtet von bedrückenden und beängstigenden Okkulterfahrungen, bei denen als Symbolisierung des Bösen ein babylonischer Dämon eine Schlüsselrolle spielt. Erst durch einen Exorzismus und die Einbindung in eine katholisch-fundamentalistische Frömmigkeit wird für Heike die Bedrohlichkeit erträglicher. Weil Heike relativ viel von ihrer Lebensgeschichte erzählt, lässt sich in ihrem Interview gut nachzeichnen, wie die dämonischen Geister, mit denen es Heike zu tun hat, entstanden sind und was sie symbolisieren. Das Interview führt von der Erzählung über Heikes bewegende Okkultsitzungen bald zurück in die Kindheit, aus der Heike eine kurze, aber dramatische Szene erzählt und damit den Ursprung ihrer lästigen Verfolger schildert:

„Die waren furchtbar hässlich, ich weiß gar nicht wie man's beschreiben soll, der Farbe nach, waren se grün, zwischen grün und verbrannt. Und die haben auch Feuer gehabt, also die waren ziemlich böse. Sie sind immer hinter mir hergegangen, sie wollten mich umbringen oder sonst was. Weiß nur eins, da war ich da hab ich geschlafen, da bin ich wach geworden, dann hab ich nach meiner Mutter gerufen, die hat mich aber nicht gehört, obwohl ich geschrien hab. Da hab ich Angst gehabt dann bin ich zur Türe, dann bin von der Türe von meinem Bett zurückgezogen worden. Von irgendetwas. Ich hab's aber nicht gesehen, was mich da gezogen hat Dann hab ich geschrien und da war ich fast am Sterben. Also, da ist meine Mutter, da hat se dann, durch's Poltern ist se dann aufmerksam geworden, dann ist se reingekommen und dann hat sie gesagt, so was ist denn mit Dir los, ich war kreidebleich, war kaum noch richtig am Leben, ich hab ziemlich schnell'nen flachen

${ }^{6}$ Die anderen beiden Typen haben wir ,Jugend forscht" (spielerisch-experimentelle Test-Praxis) und "Spiel mit dem Feuer" genannt (vgl. ebd.) Auch wenn die quantitative Verteilung der drei Typen bei den okkultpraktizierenden Jugendlichen (noch) nicht empirisch überprüft wurde, kann man davon ausgehen, dass sich im Typ "Jugend forscht" sehr wahrscheinlich die überwiegende Mehrheit der Jugendlichen findet.

Heike (Name geändert) wurde im Projekt „Wege der Entzauberung“ interviewt. Für eine ausführlichere Fallbeschreibung vgl. ebd., 98-109.

${ }^{8}$ Für die Falldarstellung Bernd (Name geändert) vgl. ebd., 110-124.
Herzschlag gehabt. Dann hab ich gesagt: ,Mama ich glaub ich sterb ich sterb, die holen mich, die holen mich!' Das klingt vielleicht ein wenig phantastisch, wie'nen Film oder so."

In der Tat klingt dies phantastisch, als wär's ein Horrorfilm oder ein Alptraum aus der Kindheit. Und genau dies ist sehr aufschlussreich. Denn hier und in anderen ähnlichen Interviewpassagen wird nachvollziehbar, aus welchem Stoff die lästigen Jenseitigen sind, die Heike symbolisiert und die sie bedrängen und die sie erst später mit Namen nennen und auch rufen und beschwören lernt. Erst nachträglich identifiziert sie den Dämon in einem Bildband, in dem sie eine Fotografie der babylonischen Statue findet, die dem lästigsten ihrer Dämonen ähnlich sieht.

Bernd (19) hat turbulente Jahre der okkulten Reiz- und Erlebnissuche als satanistischer Randalierer hinter sich. Bereits im Alter von dreizehn oder vierzehn Jahren fühlt er sich zur Waver-Szene hingezogen. Es hat ihn sehr beeindruckt, dass Wavern, die ja durch ihre Frisur sofort auffallen, auf der Straße ausgewichen wird. Der Besuch beim Friseur verhilft Bernd zu dem Privileg, in seinem Stadtteil als Waver, mit Herzklopfen zwar, aber stolz durch die Straßen zu schreiten. In der Schule erscheint Bernd alkoholisiert, malt Hakenkreuze, wirft mit Stühlen, provoziert die Lehrerin. Nach zwei Schulwechseln wird er vom Gymnasium auf die Hauptschule zurückgestuft.

Mit Freunden zusammen leiht sich Bernd satanistische Bücher in der Bibliothek, sie veranstalten nächtens Happenings in einer Leichenhalle, experimentieren mit Telepathie und versuchen sich mit ,schwarzen Messen'. Immer mehr tritt dabei die zentrale Gestalt des Teufels in Bernds Vorstellungswelt. Bernd sucht den Teufel und seine Nähe, um „Schutz von unten“ zu erlangen. Experimente mit Drogen und S-Bahn-Surfen gehören ebenso zur satanistischen Karriere Bernds wie schließlich ein unter Alkoholeinfluss und satanistischem Größenwahn begangener erpresserischer Autodiebstahl, der ihn nach der ernüchternden Erfahrung der Untersuchungshaft zur Besinnung bringt und dazu, die Hilfe eines Therapeuten in Anspruch zu nehmen.

Auf dem Hintergrund seiner Lebensgeschichte werden Traumatisierungen und Selbstspannungen, die in der okkult-satanistischen Karriere Bernds eine Rolle spielen und seine Symbolisierung des Bösen prägen, verständlicher. Im Interview erfahren wir Bernds erschütternde Geschichte: seine allein erziehende Mutter ist Alkoholikerin. Wie hinter einer Nebelwand ist sie meist unerreichbar, sie vernachlässigt das Kind, der kleine Bernd muss gelegentlich Verantwortung für seine betrunkene Mutter übernehmen, sie nicht nur ertragen, sondern wortwörtlich nach Hause oder ins Bett tragen. Aus diesem Einblick in Bernds Kindheit wird verständlicher, wie das Thema „Macht und Ohnmacht" zu Bernds Lebensthema geworden ist und sich in den verschiedenen Varianten seiner Symbolisierung des Bösen spiegelt.

Die Fälle von Heike und Bernd, wie auch andere Fallanalysen aus unserer Forschung, legen den Schluss nahe, dass in satanistischen Symbolisierungen 
und Praktiken Kindheitsängste, Traumatisierungen und Gefühle eigener Ohnmacht zum Ausdruck kommen, bearbeitet und versuchsweise kompensiert werden. Beide Fälle demonstrieren jedoch auch, dass dies nicht allein sehr riskant sein kann, sondern zuweilen - jedenfalls mittelfristig auf den betreffenden Lebensabschnitt bezogen - in Sackgassen führen kann, indem die Ängste ins Unerträgliche steigen oder die Jugendlichen mit gesellschaftlichen Normen bzw. dem Gesetz in Konflikt geraten.

\section{Die offene Frage nach der Wirkung von Symbolisierungen des Bösen}

Bei solch überaus starker subjektiver Symbolisierung wie bei Heike und Bernd treten die medialen Symbolisierungen in den Hintergrund. Doch im Blick auf die breitere Mehrheit von Jugendlichen muss auch dieser Faden wieder aufgegriffen werden. Welche Wirkung hat die Konsumierung von Symbolisierungen des Bösen in der Jugendkultur? Was bewirken satanistische Filme, Berichte über satanische Praktiken in Jugendzeitschriften oder das Genießen satanistischer Musik bei Jugendlichen, was lösen sie aus? Viele haben eine einfache Antwort zur Hand: Nachahmung. Ganz von der Hand zu weisen ist dieser Wirkmechanismus nicht. Manche Jugendlichen berichten in unseren Interviews, dass es die Thematisierung im Religionsunterricht gewesen sei, die ihre Lust aufs Praktizieren erst wachgerufen habe. Doch diese Antwort ist zu einfach. Für die medialen Symbolisierungen des Bösen könnte man, vergleichbar zur Wirkung von Gewaltdarstellungen im Film ${ }^{9}$ von einer mimetischen Wirkung eine kathartische Wirkung unterscheiden. Vergleichbar mit der mutmaßlich produktiven Wirkung des Nacherlebens von Gewalt in Märchen, ${ }^{10}$ könnte man auch eine produktive Bearbeitung von Unheimlichkeit und Ängsten mutmaßen. Die Symbolisierung des Bösen könnte hier als Weg verstanden werden, dem Unheimlichen etwas von seiner Heimlichkeit zu nehmen und damit bearbeitbar zu machen. ${ }^{11}$ Man muss allerdings dabei auch bedenken, dass diese Symbolisierung des Bösen nicht stattfindet in einem pädagogischen, meditativen oder therapeutischen Setting, sondern in einem Unterhaltungs-Setting, das auf Nervenkitzel aus ist. Und wenn die Symbolisierung des Bösen zum Adrenalin steigernden Zeitvertreib stattfindet, bleibt vermutlich auch ein möglicher kathartischer Effekt unter der Oberfläche - mit unklarem Ausgang.

9 Vgl. als neueren Beitrag Hans-Martin Gutmann, Gewalt, in: Kristian Fechtner/Gotthard FerVgl. als neueren Beitrag Hans-Martin Gutmann, Gewalt, in: Kristian Fechtner / Gotthard Fer-
mor / Uta Pohl-Patalong / Harald Schroeter-Wittke (Hg.), Handbuch Religion und Populäre Kultur, Stuttgart 2005, 112-120.

10 Vgl. Bruno Bettelheim, Kinder brauchen Märchen, Stuttgart 1975.

1 Für das Okkultpraktizieren habe ich dies, zusammen mit $W$. Helsper, besonders profiliert in: Werner Helsper / Heinz Streib, Okkultismus in der Adoleszenzkrise. Zur Durchdringung von Okkultismus, Religion und Selbstreflexivität, in: WzM 46/1994, 183-198. Vgl. auch Heinz Streib, Entzauberung der Okkultfaszination. Magisches Denken und Handeln in der Adoleszenz als Herausforderung an die Praktische Theologie, Kampen 1996.
Noch brisanter stellt sich die Frage nach den Folgewirkungen der Symbolisierung des Bösen für die subjektiven Symbolisierungen; die Antwort ist jedoch ebenso offen. Am etwas breiteren Phänomenbereich der Okkultfaszination - die jedoch vielfältig die Symbolisierung des Bösen einschließt - habe ich im Grunde genau zu dieser Frage theoretisch ${ }^{12}$ und empirisch ${ }^{13}$ gearbeitet. Dabei ist deutlich geworden, dass es durchaus Fälle gibt, die die Geister, die sie riefen, (jedenfalls zunächst) nicht mehr losgeworden sind, während andere nach einem kurzen Spiel mit dem Feuer sich anderen Dingen zuwenden und ein anderer Teil der Jugendlichen ohnehin nur spielerisch die Welt erkunden möchte. Gerade die Fälle wie Heike und Bernd sind darum interessant, weil in der biographischen Langzeitperspektive erkennbar wird, dass hier dennoch Wege der Bearbeitung gesucht und Wege der Entzauberung gefunden wurden - und dies weitgehend selbsttätig.

Damit soll nun keineswegs behauptet werden, die Frage nach den Folgewirkungen der Okkultfaszination - und besonders der Symbolisierungen des Bösen - sei zur Zufriedenheit aller lösbar und sei allenfalls eine Frage der (biographischen) Zeit. Dann könnte mein Artikel hier enden. Daraus dass die Symbolisierung des Bösen eine Ambivalenz enthält und der Ausgang je offen ist, ergeben sich Aufgaben für die pastorale und religionspädagogische Arbeit - und zur Klärung dieser Aufgaben ist es notwendig, Motive und mögliche Folgen noch einmal genauer zu analysieren.

\section{Die semantische Engführung der jugendkulturellen Symbolisierung des Bösen}

Ein erster Hinweis ist der Semantik der jugendkulturellen Symbolisierungen des Bösen zu entnehmen. Bei näherem Zusehen fällt auf, dass personale Symbole im Vordergrund stehen: Symbolisierungen des Bösen als auf das Individuum bezogene Personifizierungen. Diese haben die Gestalt anthropomorpher Wesen (teils mit zoo-morphen Anteilen): Teufel, Dämonen, böse Geister. Diese Wesen sind darum unheimlich und bedrohlich, weil als ihr Zuhause eine dunkle Hinterwelt vorgestellt wird, aus der sie, besonders wenn man sie ruft oder beschwört, oder vielleicht auch bereits, wenn man ihren Namen ausspricht, jederzeit und unberechenbar ins Diesseits eingreifen, um das persönliche Schicksal negativ zu beeinflussen.

Aufmerksames Studium der vielfältigen Erzählungen der Jugendlichen zeigt, wie stark ihre Symbolisierungen des Bösen charakterisiert sind durch solche Personifizierung und Individualisierung und wie wenig darüber hinaus etwa das Schicksal der Menschen und der Menschenwelt mit-symbolisiert wird. Andere semantische Felder, die mit dem Bösen assoziiert sind - Krank-

12 H. Streib, Entzauberung (Anm. 11).

13 H. Streib / A. Schöll (Anm. 5). 
heit und Katastrophen, das Böse als kontingente Schicksalsmacht, Unterdrückung und Krieg oder Massenvernichtung - liegen weitgehend außerhalb des semantischen Feldes der jugendkulturellen Symbolisierung des Bösen.

Doch ist in dieser semantischen Fokussierung auf Personifizierung und individuelle Betroffenheit eine genauere Antwort auf die Frage vorbereitet, was denn eigentlich hier symbolisiert und bearbeitet wird. Die folgende psychoanalytische Betrachtung kann daran anschließen.

\section{Psychoanalytische Betrachtung der jugendkulturellen Symbolisierung des Bösen}

Die Adoleszenz ist eine besondere Zeit der Auseinandersetzung mit der interpersonalen Dynamik des Nahbereichs - in Gestalt der Auseinandersetzung mit und eventuell Ablösung von den Eltern bzw. den inneren Bildern, die sich von den Eltern eingeprägt haben. Der besonders virulenten Auseinandersetzung mit den dominanten Elternbildern wegen kann die Adoleszenz als Phase der „zweiten Individuation“ bezeichnet werden. ${ }^{14}$ Wenn man zudem davon ausgeht, dass in der Adoleszenz nicht nur die „guten“ Anteile der Elternbilder, sondern auch die „bösen“ Anteile zur Bearbeitung anstehen, dann ist es ein kleiner Schritt, die Beschäftigung mit bösen Geistern, Dämonen und dem Teufel zu verstehen. Die Symbolisierung des Bösen könnte so eine psychoanalytische Deutung finden.

Aus unseren Interviews mit Jugendlichen - besonders gut ist dies im Interview mit Heike nachvollziehbar - wird erkennbar, dass in Okkulterfahrungen Kindheitsängste zutage treten und wie sich aus den damals bedrängenden Gestalten die Geister entwickeln. Vielen okkultfaszinierten Jugendlichen ist solche Kontinuität der angsterregenden jenseitigen Gestalten von der frühen Kindheit bis ins Jugendalter nicht bewusst; dennoch kann die Behauptung gewagt werden, dass die Geister unter dem Glas und die dämonischen Jenseitigen und besonders Satan eben dies sind: Symbolisierungen des eigenen Unheimlichen.

Dieser Gedankengang findet Bestätigung bei Freud. Ausdrücklich entwickelt er aus einer Art Wortspiel die These, dass „das Unheimliche“ als „das heimlich Eigene" $\mathrm{zu}$ verstehen ist. ${ }^{15}$ Der Teufel gehört für Freud ebenso wie die Dämonen und Geister zu den privaten Symbolsystemen, in denen die eigenen Ängste und Schatten zum Ausdruck kommen. Was den Inhalt des Teufelssymbols ausmacht, entfaltet Freud anhand des Malers Haizmann, dem Fall einer "Teufelsneurose aus dem 17. Jh.“: 16

14 Vgl. Peter Blos, The Second Individuation Process of Adolescence, in: The Psychoanalytic Study of the Child 22/1967, 162-186.

15 Vgl. Sigmund Freud, Das Unheimliche, in: Gesammelte Werke, Bd. 12, London 1919, $227-$ 268.

16 Sigmund Freud, Eine Teufelsneurose im 17. Jahrhundert, in: Gesammelte Werke, Bd.13, Frankfurt a.M. 1923, 317-353.
Wenn der Vater, davon geht Freud aus, „das individuelle Urbild sowohl Gottes wie des Teufels" ist, ${ }^{17}$ dann ist über den Teufel genauer zu sagen, dass in seinem Bild - als zum Dämon abgespaltener oder "gefallener" (Vater-)Gott - nur mehr die bösen Anteile vereinigt sind.

Was folgt aus dieser psychoanalytischen Deutung? Wenn man mit Erikson die adoleszente Arbeit an der Identität als Bearbeitung der Kindheitsmuster begreift, könnte auch die Bearbeitung der Kindheitsängste und bedrohlichen Elternfiguren, sofern sie erfolgreich verläuft, als Teil der Identitätsbildung begriffen werden. Es ist von daher zumindest denkbar, die Symbolisierungen des Bösen in der adoleszenten Psychodynamik als Teil der Identitätsbildung zu verstehen und ggf. auch das Modell der "Regression im Dienste des Ichs“ als Interpretationsmuster zu erwägen. ${ }^{18}$ Denkbar ist, wenn wir Erikson folgen jedoch auch eine nicht erfolgreiche Lösung der Identitätskrise in der Adoleszenz und die Ausbildung einer negativen Identität, die in der Identifizierung mit dem Aggressor, der Identifikation mit dem Bösen entstehen und fortbestehen kann. Der Ausgang der Symbolisierung des Bösen in der Adoleszenz kann also keineswegs als gesichert gelten. Die Aufgaben für Seelsorge und Religionspädagogik werden präziser.

In der analytischen Deutung der jugendkulturellen Symbolisierung des Bösen, wie wir diese soweit entfaltet haben, wurde in erster Linie eine lebensgeschichtlich rückwärts gewandte Perspektive eingenommen. Mit Ricoeur gesprochen, ${ }^{19}$ stand dabei die archäologische Bedeutung des Symbols im Vordergrund. Diese muss - dies ist trotz aller Risiken der Identitätsbildung in der Adoleszenz festzuhalten - durchaus nicht in eine lebensgeschichtliche Sackgasse, in Stagnation führen, sondern kann Zukunft eröffnen. Und nicht immer brauchen Jugendliche dabei Hilfe von außen. Dennoch wird bereits erkennbar, dass hierbei seelsorgerliche und religionspädagogische Begleitung hilfreich sein kann und diese Hilfe idealiter darin bestehen könnte, diesen psychodynamisch-archäologischen Bearbeitungsprozess für die biographische Selbstreflexion der Jugendlichen zu erschließen und verstehbar zu machen.

Dennoch - und hier kann man viel gewinnen, wenn man mit Ricoeur weiterdenkt - ist von einer zweiten, teleologischen Bedeutungsdimension der Symbole zu sprechen. Dementsprechend wäre nun nach der Zukunftsbedeutung auch und gerade der Symbole des Bösen zu fragen. Dass dies nicht ohne kritischen Bezug auf die Symbolisierungen des Bösen in der Jugendkultur geht, liegt auf der Hand. Die nun folgenden symbolkritischen Betrachtungen nehmen einige Grundgedanken aus der Symboltheorie und Religionsphilosophie Tillichs auf, fokussieren jedoch auf einen formalen semiologisch-hermeneutischen und einen materialen semantischen Aspekt.

\footnotetext{
Ebd., 332.

18 Blos (ebd., 173) weist ausdrücklich auf progressive Resultate von „Regression“ hin.
}

Vgl. Paul Ricoeur, Freud and Philosophy. An Essay on Interpretation, New Haven, 1965 


\section{Semiologisch-hermeneutische Stil-Unterschiede im Verstehen des Bösen}

Symbole weisen über sich hinaus, auf etwas anderes hin. Ein philosophisch und theologisch angemessener Symbolbegriff ist darum strukturiert durch die Figur des Über-sich-Hinausweisens, wie dies klassisch in Tillichs ${ }^{20}$ grundlegendem Merkmal der "Uneigentlichkeit“ formuliert wird (und die Offenheit von Tillichs Symbolbegriff für eine semiotische Reformulierung kennzeichnet). Dass ein solch reifes Symbolverständnis nicht jedem Menschen geschenkt ist, sondern hier eine ganze Menge zu lehren und lernen ist, dass hier Entwicklungsschritte angeregt und unternommen werden müssen, ist offenkundig - nicht zuletzt angesichts der jugendkulturellen Symbolisierungen des Bösen. Die Bestimmung des Lern- und Entwicklungsziels für das Symbolverstehen kann sich an Tillichs Symboltheorie anschließen, ja sich als entwicklungspsychologische Übersetzung seiner Symboltheorie verstehen; ${ }^{21}$ für die kleinschrittige Modellierung der Entwicklung von Religion und Symbolverstehen maßgebend ist die faith development theory Fowlers $^{22}$ - auch und gerade in ihrer Offenheit für Weiterentwicklungen und Modifikationen..$^{23}$

Ein „reifes" Symbolverständnis, das mit der semiotischen Differenz arbeitet, gehört auf alle Fälle zum dialogischen Stil (Streib) bzw. conjunctive faith der Stufe 5 (Fowler); jedoch ist das Bewusstsein einer Symboldifferenz bereits auf Stufe 4 des individuative-reflective faith (Fowler) bzw. des systemischen Stils (Streib) ausgebildet und auf Stufe 3 angelegt. Dieses für die religiöse Entwicklung entworfene Modell ist auch für die Einordnung der hermeneutischen Stile aufschlussreich, die wir in der Symbolisierung des Bösen antreffen. Hier können und sollten unterschieden werden:

- die dinghafte, konkrete - und im Grunde nicht-symbolische - Vorstellung des Bösen als angstbesetzte konkrete Wesen einer Hinterwelt, die in der Entwicklungs-Hierarchie weiter unten stehen und den ersten beiden Stufen bzw. Stilen angehören,

${ }^{20}$ Vgl. Paul Tillich, Das religiöse Symbol, in: Main Works/Hauptwerke, Bd. 4, Berlin / New York $1928,213-228$

21 Anders als theoretisch (philosophisch, semiotisch, theologisch) ist angesichts der Plausibilitätsprobleme strukturgenetischer Theorieannahmen, besonders was die Transformation auf höhere Stufen angeht, Entwicklung von Religion und Symbolverstehen nicht zu begründen (Vgl. Heinz Streib, Kulturdiagnostische Aspekte von Tillichs Symboltheorie, in: Werner Schüssler / Christian Danz / Erdmann Sturm, Internationales Jahrbuch für die Tillich-Forschung, Band 2, (in Arbeit).

${ }_{22}$ James W. Fowler, Stages of Faith. The Psychology of Human Development and the Quest for Meaning, San Francisco 1981

${ }_{23}$ Heinz Streib, Faith Development Theory Revisited: The Religious Styles Perspective, in: International Journal for the Psychology of Religion 11/2001, 143-158; ders., Faith Development Research Revisited: Accounting for Diversity in Structure, Content, and Narrativity of Faith in: International Journal for the Psychology of Religion 15/2005, 99-121.
- reflexive Unterscheidung von Symbol und Symbolisiertem und damit erste Schritte der "entzaubernden“ analytischen Aufarbeitung des symbolisierten Bösen, die besonders Stufe/Stil 4 charakterisiert und ein wünschbares Zwischenziel (Bildungsziel) sein kann,

- und schließlich ein Symbolverständnis, das nicht mehr nur „entmythologisierend" und analytisch entlarvend vorgeht, sondern bei vollem Bewusstsein der semiotischen Differenz die welterschließende Tiefen-Bedeutung der Symbole - auch der Symbole des Bösen - würdigt und gelten lässt.

Ein wichtiges Ziel religionspädagogischer und pastoraler Arbeit jedenfalls lässt sich aus diesen Überlegungen herleiten, nämlich auf ein Symbolverständnis hinzuarbeiten, das mit der semiotischen Differenz operiert und daher den Teufel und die Dämonen als Symbol verstehen kann und somit entzaubert.

\section{Semantische Symbolkritik an der Symbolisierung des Bösen in der Jugendkultur}

Eine zweite symbolkritische Überlegung schließt an die oben dargestellte semantische Engführung der Symbolisierung des Bösen in der Jugendkultur an und sucht eine semantische Erweiterung zu begründen. Tillichs Begriff des Dämonischen ${ }^{24}$ ist insofern erhellend für unsere Überlegung, als durch die polare Unterscheidung von Göttlichem und Dämonischem religionsphilosophisch angemessene Gravitationspunkte ins semantische Feld eingezeichnet werden. Das Dämonische ist dann zu definieren als Bedingtes, das sich an die Stelle des Unbedingten setzt. Semantisch ist damit das Dämonische enorm erweitert und umfasst auch kapitalismus-, faschismus-, und selbst kircheninhärente Dämonie. Religionsphilosophisch und systematisch-theologisch ist es sinnvoll, die Symbolisierungen des Bösen mit diesem Begriff des Dämonischen zu interpretieren.

Symbolisierungen des Bösen in der Jugendkultur, das wäre dann der Anspruch, sollten aus der oben skizzierten semantischen Engführung herausgeholt werden. Dies eröffnet den Blick auf lohnende, aber schwierige pastorale und religionsdidaktische Aufgaben. Das Problem ist die Vermittlung; denn es betrifft einen Symbolkonflikt, der nicht schlicht kognitiv-diskursiv durch einen Wettbewerb von Geltungsansprüchen entschieden wird, sondern mit tiefen psychodynamisch und lebensgeschichtlich verwurzelten Prägungen (Ängsten, Traumata, Selbstspannungen) zu tun hat. So schwierig diese Aufgabe ist, aussichtslos ist sie nicht. Im Bewusstsein der skizzierten Tiefendimen-

${ }^{24}$ Vgl. Paul Tillich, Religionsphilosophie, in: Main Works / Hauptwerke, Bd. 4, Berlin / New York 1925, 117-170; ders., Das Dämonische. Ein Beitrag zur Sinndeutung der Geschichte, in Main Works / Hauptwerke Bd. 5, Berlin / New York 1926, 99-123; ders., Der Begriff des Dämonischen und seine Bedeutung für die Systematische Theologie, in: Gesammelte Werke, Bd. VIII, Stuttgart 1926, 285-291. 
sionen können den Jugendlichen Brücken gebaut werden für eine semantische Erweiterung der Symbolisierung des Bösen, die aus der personalistisch-individualistischen Engführung herausführt.

\section{Ausblick auf die pastorale und religionspädagogische Praxis}

Hinweise für pastorale und religionspädagogische Arbeit habe ich bereits einige notiert. Hier fasse ich zusammen: Es gilt,

- sich möglichst eng an die biographische Entwicklungsdynamik der Jugendlichen anzuschmiegen

- die Symbolisierungen des Bösen in der adoleszenten Psychodynamik als Teil der Identitätsbildung zu verstehen,

- den psychodynamisch-archäologischen Bearbeitungsprozess, der im Umgang mit den Symbolisierungen des Bösen stattfindet, für die biographische Selbstreflexion der Jugendlichen zu erschließen und verstehbar zu machen,

- auf ein Symbolverständnis hinzuarbeiten, das mit der semiotischen Differenz operiert und daher den Teufel und die Dämonen als Symbol verstehen kann und somit entzaubert,

- eine semantische Erweiterung der Symbolisierung des Bösen anzuregen und zu befördern, die aus der personalistisch-individualistischen Engführung herausführt und sich von einem theologischen Begriff des Dämonischen, wie ihn Tillich entfaltet hat, anregen lässt.

Als Fazit im Blick auf die praktische Arbeit mit Jugendlichen und ihren Symbolisierungen des Bösen kristallisiert sich ein seelsorgerliches, auf die Lebensgeschichte der Jugendlichen bezogenes Profil heraus. Dies hat seinen Platz in seelsorgerlicher Beratung und diese Perspektive kann leitend sein für Gruppengespräche, etwa im Rahmen der Jugendarbeit. Auch für die Religionspädagogik, speziell die Symboldidaktik, die sich als religionsdidaktischer Königsweg hier anbietet, ist eine entsprechende Profilierung vorzuschlagen: Symboldidaktik sollte dafür über eine kritische Symbolkunde hinaus auf die erfahrungsorientierte, subjektorientierte Thematisierung der Symbolisierungen der Jugendlichen zielen, um den Prozess der Entzauberung voranzubringen.

Heinz Streib (Ph. D.), Jahrgang 1951, ist Professor für Evangelische Theologie und ihre Didaktik/Religionspädagogik und ökumenische Theologie am Research Center for Biographical Studies, Universität Bielefeld; er leitet die Forschungsstelle Biographische Religionsforschung.

Postfach 1001 31, 33501 Bielefeld

E-Mail: heinz.streib@uni-bielefeld.de 